\title{
Highly stable magic angle spinning spherical rotors
}

\author{
Thomas M. Osborn Popp, Alexander Däpp, Chukun Gao, Pin-Hui Chen, Lauren E. Price, \\ Nicholas H. Alaniva, and Alexander B. Barnes
}

Laboratory for Physical Chemistry, ETH Zürich, Vladimir-Prelog-Weg 2, 8093 Zürich, Switzerland

Correspondence: Alexander Barnes (alexander.barnes@phys.chem.ethz.ch)

Received: 30 January 2020 - Discussion started: 1 April 2020

Revised: 7 May 2020 - Accepted: 11 May 2020 - Published: 18 June 2020

\begin{abstract}
The use of spherical rotors for magic angle spinning offers a number of advantages, including improved sample exchange, efficient microwave coupling for dynamic nuclear polarization nuclear magnetic resonance (NMR) experiments, and, most significantly, high frequency and stable spinning with minimal risk of rotor crash. Here we demonstrate the simple retrofitting of a commercial NMR probe with MAS spheres for solid-state NMR. We analyze a series of turbine groove geometries to investigate the importance of the rotor surface for spinning performance. Of note, rotors lacking any surface modification spin rapidly and stably even without feedback control. The high stability of a spherical rotor about the magic angle is shown to be dependent on its inertia tensor rather than the presence of turbine grooves.
\end{abstract}

\section{Introduction}

Magic angle spinning (MAS) nuclear magnetic resonance (NMR) is usually used for high-resolution analysis of the local chemical environments of nuclear spins within biomolecular and inorganic solids (Schaefer and Stejskal, 1976; McDermott, 2009; Doty and Ellis, 1981; Knight et al., 2012; Retel et al., 2017; Theint et al., 2017; Petkova et al., 2005; Kong et al., 2013; Wang et al., 2013; Cegelski et al., 2002; Bougault et al., 2019; Clauss et al., 1993; Trebosc et al., 2005; Lesage et al., 2008). The sample is spun rapidly about an axis inclined at the magic angle, which is $54.74^{\circ}$ with respect to the external magnetic field $B_{0}$. This averages terms in the NMR Hamiltonian whose orientational dependence is described by the second-order Legendre polynomial $3 \cos ^{2} \theta-1$ (Andrew et al., 1959; Lowe, 1959; Andrew, 1981). For spins-1/2, MAS can yield spectra with highly resolved isotropic chemical shifts.

MAS has traditionally been performed by spinning a cylindrical rotor within a stator installed at the magic angle, thereby requiring a gas bearing to stabilize the rotor and drive gas to apply torque (Andrew, 1981; Doty and Ellis, 1981; Wilhelm et al., 2015). However, we recently showed that it is possible to spin samples via a different paradigm, namely using spherical rotors spun using a single gas stream for both the bearing and drive (Chen et al., 2018; Gao et al., 2019). This approach allows highly stable rotor spinning about a single axis inclined at the magic angle, with record rates as high as $4.6 \mathrm{kHz}\left(\mathrm{N}_{2}, 4.1 \mathrm{bar}\right)$ and $10.6 \mathrm{kHz}(\mathrm{He}, 11 \mathrm{bar})$ for $9.5 \mathrm{~mm}$ diameter rotors and $11.4 \mathrm{kHz}\left(\mathrm{N}_{2}, 3.1 \mathrm{bar}\right)$ and $28 \mathrm{kHz}(\mathrm{He}$, $7.6 \mathrm{bar}$ ) for $4 \mathrm{~mm}$ diameter rotors. Decreasing the rotor diameter permits even higher spinning rates. Additional benefits of spherical rotors include easy sample exchange and improved microwave access for dynamic nuclear polarization (DNP)-NMR experiments, yielding improved microwave $B_{1}$ field strength and homogeneity compared with current methods (Chen et al., 2019; Gao et al., 2019).

In order to make spherical rotors robust and accessible for magnetic resonance experiments and to design apparatuses capable of achieving very high MAS rates, we examined the spinning and stabilization mechanisms of these spherical rotors. Here we (i) demonstrate how a stator for spinning spheres can be easily integrated into a commercial NMR probehead and (ii) examine the spinning behavior of a series of spherical rotors with various turbine groove geometries. We show that the spinning performance of spherical rotors can be improved by using a turbine groove geometry similarly to the drive tips used in conventional cylindrical rotor MAS systems, which are themselves based on the Pelton impulse turbine (Wilhelm et al., 2015). However, we also 
find across a wide array of turbine styles that spinning performance is remarkably indifferent to the surface design and that even a rotor without turbine grooves can achieve stable, on-axis spinning. We show that a spherical rotor attains its stability from its inherent shape and mass distribution (i.e., its inertia tensor) and that turbine grooves are not essential for stable spinning.

\section{Experimental apparatus}

The experimental apparatus is depicted in Fig. 1. The stator employed for spinning spherical rotors was designed to adapt into a double-resonance APEX-style Chemagnetics probe (built decades ago for spinning $7 \mathrm{~mm}$ diameter cylindrical rotors) and 3D printed in clear acrylonitrile-butadiene-styrene (ABS) using either a ProJet MJP2500 3D printer (3D Systems, Rock Hill, SC, US) or a Form3 3D printer (Formlabs, Somerville, MA, US). A double-saddle coil made from $1.5 \mathrm{~mm}$ silver-coated copper magnet wire was wound by hand using a mandrel and wrapped in Teflon tape for insulation with the leads soldered into place in the existing radio frequency (RF) circuit of the Chemagnetics probe. The two optical fibers of the tachometer system were introduced into holes at the bottom of the semi-transparent stator. The transceiver of the original tachometer system was replaced with a more sensitive circuit. The transmitter in this circuit was an SFH756 light-emitting diode fed with $42 \mathrm{~mA}$ of current. The detector comprised an SFH250 photodiode and a transimpedance amplifier with a $4.7 \mathrm{M} \Omega$ feedback resistor followed by a gain block providing a voltage gain of 42 . The magic angle adjustment was achieved by coupling the stator to the existing angle adjust rod in the probe.

NMR experiments were performed at $7.05 \mathrm{~T}$ using a Bruker Avance III spectrometer (Bruker Corp., Billerica, MA, US). ${ }^{79} \mathrm{Br}$ spectra were taken at a transmitter frequency of $75.46 \mathrm{MHz}$ and a MAS rate of $3.5 \mathrm{kHz}$. The implementation of a double-saddle coil within the probe enabled the application of a $35 \mathrm{kHz} \mathrm{B}$ field to ${ }^{79} \mathrm{Br}$ with $300 \mathrm{~W}$ incident RF power, a significant improvement over our previous implementation of $9.5 \mathrm{~mm}$ spherical rotors, which achieved a $\mathrm{B}_{1}$ field of only $12.5 \mathrm{kHz}$ using a split coil and $800 \mathrm{~W}$ incident RF power (Chen et al., 2018).

The $9.5 \mathrm{~mm}$ spherical rotors were machined from yttriastabilized zirconia (O'Keefe Ceramics, Woodland Park, CO, US). Seven new spherical rotor designs were introduced (Fig. 2), each with a $2.54 \mathrm{~mm}$ inner diameter cylindrical through hole: notched (rotors A, C), Pelton-style (rotors B, F), circular (rotors $\mathrm{C}, \mathrm{G}$ ), dimpled (rotor D), and with no flutes (rotor H). For the notched, circular, and Pelton-style rotors, two variations were machined differing by $0.5 \mathrm{~mm}$ in depth. For spin testing, each rotor was filled with a rigid 3D printed cylindrical ABS blank terminated with $4.75 \mathrm{~mm}$ spherical radius contoured ends. For the NMR experiment, the rotor was packed with $97.1 \mathrm{mg} \mathrm{KBr}$ powder and sealed at each end with 3D printed ABS plugs. The same stator design was used for all spin testing experiments.

\section{Results and discussion}

Spherical rotors spin within the hemispherical stator cup by the application of a gas stream along the rotor's equator from a converging nozzle tangent to the rotor surface (Chen et al., 2018). The nozzle aperture is placed at the complement of the magic angle $\left(35.3^{\circ}\right)$ in order to tilt the spinning axis of the rotor to a value near the magic angle (as shown in Fig. 1c). The rotor turbine grooves are intended to provide a means to efficiently couple the gas stream to the rotor surface, converting the kinetic energy of the fluid flow into rotational kinetic energy.

The designs depicted in Fig. 2 were chosen to explore this concept. Intuition might suggest that deeper grooves allow for greater coupling to the gas stream due to the increased surface area perpendicular to the direction of fluid flow. However, the deep-grooved rotors E, F, and G could not spin stably under any of the conditions tested. It is possible that the large spaces created by the removal of material to make these deep grooves allow chaotic and turbulent flows to develop within the stator cup. The shallow-grooved rotors A, B, C, and $\mathrm{D}$, as well as rotor $\mathrm{H}$, achieved stable spinning. Figure $3 \mathrm{a}$ shows the spin test data in air at gauge pressures ranging from 0 to 4.3 bar for these five rotors. Across the five turbine geometries, the spinning rate increased non-linearly with respect to the applied pressure. The maximum spinning rates for the five rotors at the pressures tested ranged between 4 and $6 \mathrm{kHz}$ in air, with Pelton-style rotor B having the highest maximum spinning rate of $5.7 \mathrm{kHz}$. Note that the maximum tested pressure was not limited by the rotors, as their spinning rates would be predicted to increase with increasing pressure, but rather the maximum pressure was limited to $\sim 4$ bar due to safety concerns with regard to the connecting assemblies. Rotor B's maximum observed rate was a significant improvement over our previous maximum of $4.6 \mathrm{kHz}$ for $9.5 \mathrm{~mm}$ rotors in air, which was achieved using rotor $\mathrm{A}$ at $4.1 \mathrm{bar}$ (Chen et al., 2018). As rotor A also performed better in our current tests, with a maximum rate of $5.2 \mathrm{kHz}$ at $4.1 \mathrm{bar}$, we attribute some of the performance gains to the higher-precision 3D printing of our latest stators. However, the further increase to $5.7 \mathrm{kHz}$ with rotor B is likely to be due to the Pelton-style grooves more efficiently coupling to the fluid flow.

A significant finding was that rotor $\mathrm{H}$, with its smooth surface and lack of grooves, spun stably and on-axis. While not delivering the highest-frequency spinning of the tested rotors, its performance was comparable to many of the designs with machined grooves. Figure $3 \mathrm{~b}$ shows the ${ }^{79} \mathrm{Br}$ spectrum of $\mathrm{KBr}$ at $3.5 \mathrm{kHz}$ MAS using rotor $\mathrm{H}$. The stator's pitch angle was adjusted until the rotor's spinning axis was inclined to the magic angle, as observed by measuring the decay of rotor echoes out to $10 \mathrm{~ms}$ in the time domain data. The spec- 


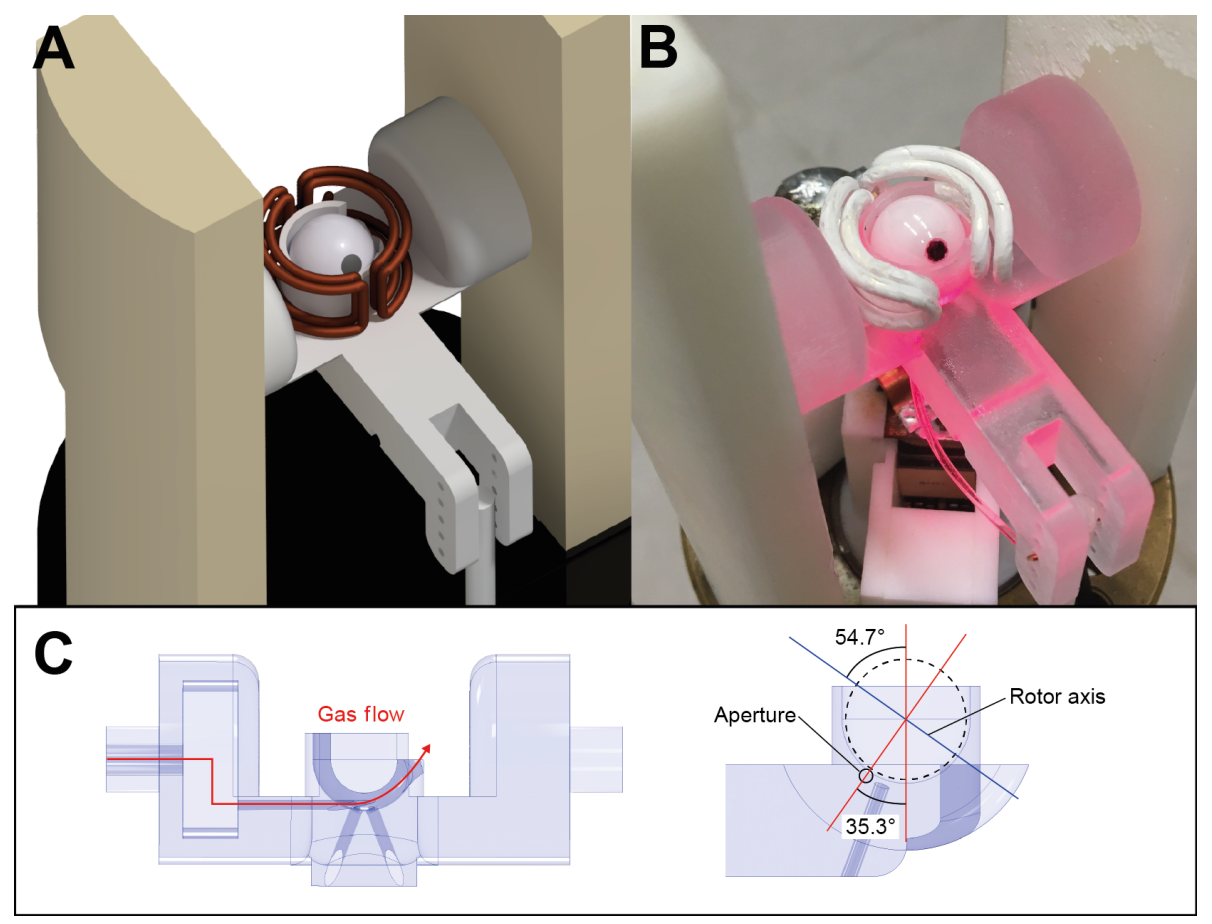

Figure 1. Experimental apparatus. (a) Probehead design schematic depicting the stator with a double-saddle coil, spherical rotor, and angle adjust arm. (b) Fully assembled probehead. (c) Cross-section views of the stator showing the drive gas inlet and angled through holes for the fiber optic tachometer. Drive gas is fed through the nozzle aperture placed at the complement of the magic angle $\left(35.3^{\circ}\right)$ into the stator cup.
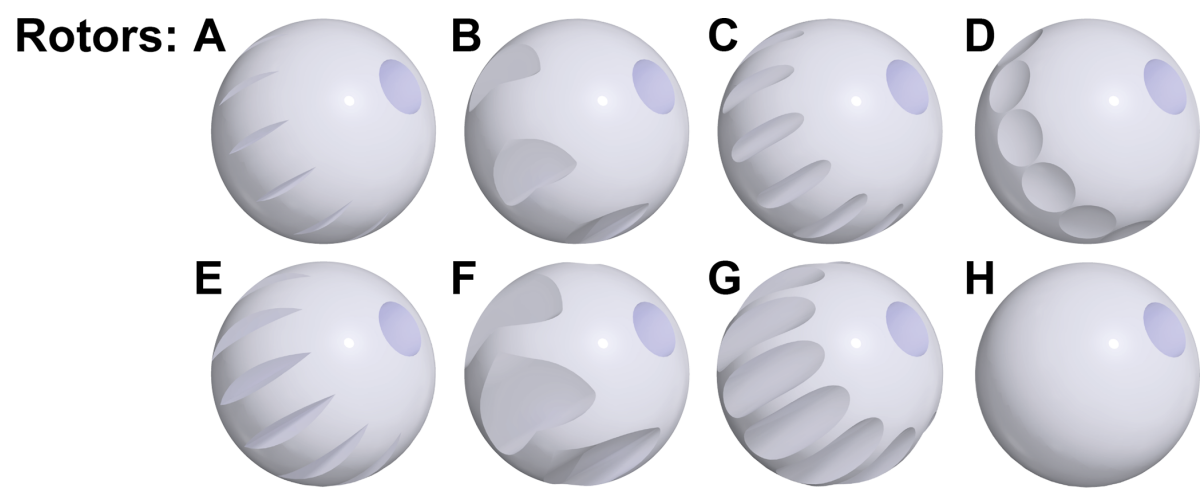

Figure 2. $9.5 \mathrm{~mm}$ spherical rotor turbine groove designs. Each rotor has a $2.54 \mathrm{~mm}$ diameter through hole. A: $1260^{\circ}$ notched grooves, $0.35 \mathrm{~mm}$ depth (as described previously by Chen et al., 2018). B: 6 impeller-style grooves, $0.25 \mathrm{~mm}$ radius. C: 12 circular $0.25 \mathrm{~mm}$ radius grooves. D: 12 dimpled grooves, $4.75 \mathrm{~mm}$ radius dimples. E: $1260^{\circ}$ notched grooves, $0.85 \mathrm{~mm}$ depth. F: 6 impeller-style grooves, $0.5 \mathrm{~mm}$ radius. G: 12 circular $0.5 \mathrm{~mm}$ radius grooves. $\mathrm{H}$ : smooth surface, no flutes machined.

trum shows the spinning sideband manifold equally spaced by $3.5 \mathrm{kHz}$, corroborating the spinning rate observed by optical tachometry. The spinning was stable, with a standard deviation of $\pm 1 \mathrm{~Hz}$ without the use of any spinning regulation mechanism (Fig. 3c). The ability of rotor $\mathrm{H}$ to spin stably at reasonable MAS rates implies that while turbine grooves can improve fluid flow and rotor coupling, a significant contribution to the overall spherical rotor spinning mechanism is simply from the torque created by drag induced by the driving gas stream moving across the rotor surface. Additionally, the fact that rotor $\mathrm{H}$ established a stable spinning axis about its own axis of symmetry shows that the grooves do not direct the rotor to spin about this axis, but rather that the geometry of the rotor itself must be responsible.

A spherical rotor with a cylindrical through hole is a solid known as a spherical ring. The moments of inertia for a spherical ring of constant density $\rho$, outer radius $R$, and inner radius $r$, where $R \geq r$ and $z$ lies along the axis of symmetry, 

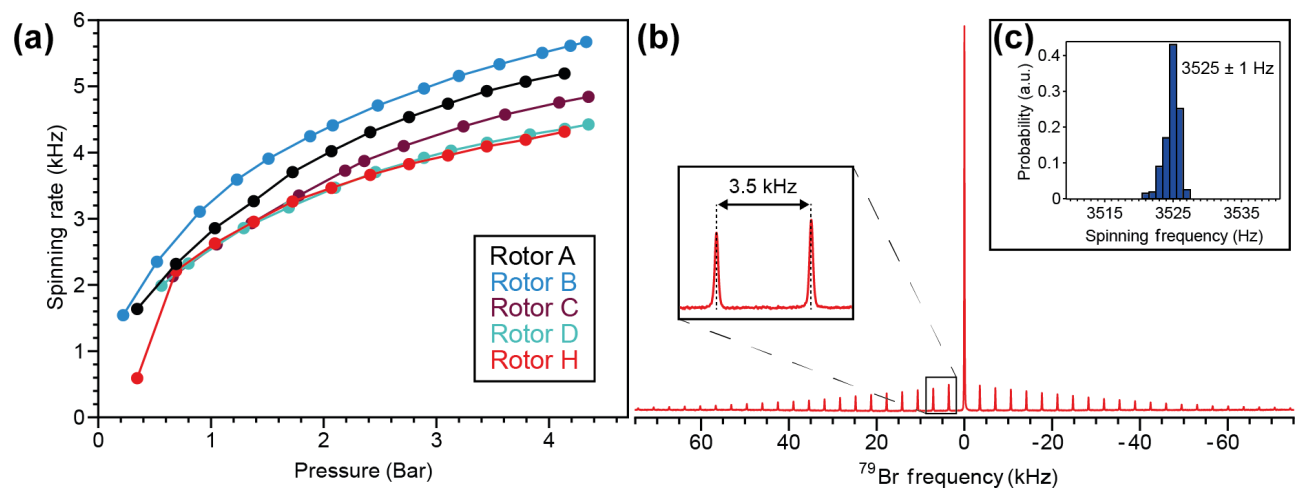

Figure 3. Spin test data. (a) Spinning rate as a function of applied air pressure for rotors A, B, C, D, and $\mathrm{H}$. (b) ${ }^{79} \mathrm{Br}$ spectrum of $\mathrm{KBr}$ packed into rotor $\mathrm{H}$ and spinning at $3.5 \mathrm{kHz}$ at the magic angle; 512 scans. (c) Histogram of spinning frequencies without spinning regulation over the $10 \mathrm{~min} \mathrm{KBr}$ data acquisition period.

are given by

$$
\begin{aligned}
& I_{x}^{\mathrm{sr}}=I_{y}^{\mathrm{sr}}=\frac{4}{15} \pi \rho\left(R^{2}-r^{2}\right)^{3 / 2}\left(\frac{4 R^{2}+r^{2}}{2}\right), \\
& I_{z}^{\mathrm{sr}}=\frac{4}{15} \pi \rho\left(R^{2}-r^{2}\right)^{3 / 2}\left(2 R^{2}+3 r^{2}\right) .
\end{aligned}
$$

As most previous MAS experiments have been performed with cylindrical rotors, it is worth examining the inertia tensor of a cylindrical shell for comparison. For a cylindrical shell of constant density $\rho$, outer radius $R$, inner radius $r$, and length of $2 k R$, where $k$ is the aspect ratio and $R \geq r$, the moments of inertia are given by

$$
\begin{aligned}
& I_{x}^{\mathrm{cs}}=I_{y}^{\mathrm{cs}}=\pi \rho k R\left(R^{2}-r^{2}\right)\left(\frac{(2 k+1) R^{2}+r^{2}}{2}\right), \\
& I_{z}^{\mathrm{cs}}=\pi \rho k R\left(R^{2}-r^{2}\right)\left(R^{2}+r^{2}\right) .
\end{aligned}
$$

Figure 4 shows the magnitudes of the moments of inertia for a spherical ring and a cylindrical shell $(k=4)$ as a function of the inner radius given by Eqs. (1) and (2). For the spherical ring, $I_{z}$ is greater than or equal to the transverse moments $I_{x, y}$ for all values of $r$, while for the cylindrical shell, $I_{z}$ is less than or equal to the transverse moments $I_{x, y}$ for all values of $r$. When $r=0$, the moments of inertia for the spherical ring and cylindrical shell are equivalent to those of a solid sphere and solid cylinder, respectively. Note that while Fig. $4 \mathrm{~b}$ is representative of the high-aspect-ratio cylindrical rotors commonly used in MAS experiments, when $k$ is low such that the geometry is disk-like, $I_{z}$ will be greater than $I_{x, y}$ for all values of $r$.

Consider a rigid object with principal moments of inertia $I_{z}, I_{y}$, and $I_{x}$, rotating about the axis coincident with $I_{z}$ with a constant angular velocity vector of $\left(\omega_{z}, 0,0\right)$. If a small perturbation is applied, the angular velocity vector assumes the form $\left(\omega_{z}, \lambda, \mu\right)$. Through substitution of this angular velocity vector into Euler's equations to yield a second-order linear differential equation with respect to $\lambda$ (Thornton and
Marion, 2004; Narayanan et al., 2008), we arrive at

$\ddot{\lambda}+\frac{\left(I_{z}-I_{x}\right)\left(I_{z}-I_{y}\right)}{I_{y} I_{x}} \omega_{z}^{2} \lambda=0$.

Equation (5) can be used to arrive at the famous result of the intermediate axis theorem, which states that objects with $I_{z}>I_{y}>I_{x}$ will spin stably only about the first and third principal axes ( $z$ and $x$ ), while a rotation about the second principal axis $(y)$ is unstable. However, for an axially symmetric object, where the axis of symmetry is aligned with the $z$ axis and $I_{y}=I_{x}$ (such as a spherical ring or a cylindrical shell), Eq. (3) becomes

$\ddot{\lambda}+\frac{\left(I_{z}-I_{x}\right)^{2}}{I_{x}^{2}} \omega_{z}^{2} \lambda=0$.

Equation (6) implies that a rotation about the axis of symmetry $(z)$ is always stable, as

$\frac{\left(I_{z}-I_{x}\right)^{2}}{I_{x}^{2}} \omega_{z}^{2}>0$.

If, however, the $x$ axis is instead the axis of symmetry and $I_{z}=I_{y}$, Eq. (5) reduces to $\ddot{\lambda}=0$, whose solutions grow linearly in time. Thus, rotation about an axis perpendicular to the axis of symmetry is unstable. According to these results, we should expect that a rotation about the axis of symmetry $(z)$ is stable and a rotation perpendicular to the axis of symmetry is unstable, regardless of whether $I_{z}$ is greater than or equal to $I_{x}$.

From this result, we might expect that a spherical shell (axis of symmetry is the highest inertia axis) and a cylindrical shell (axis of symmetry is the lowest inertia axis) to both be continuously stable during a spin about their axis of symmetry. However, it has been observed experimentally that when avenues to dissipate energy are present, an axially symmetric object can dissipate energy to end up rotating about the axis that minimizes its rotational kinetic energy for a fixed angular momentum, which is the axis with the largest moment of 

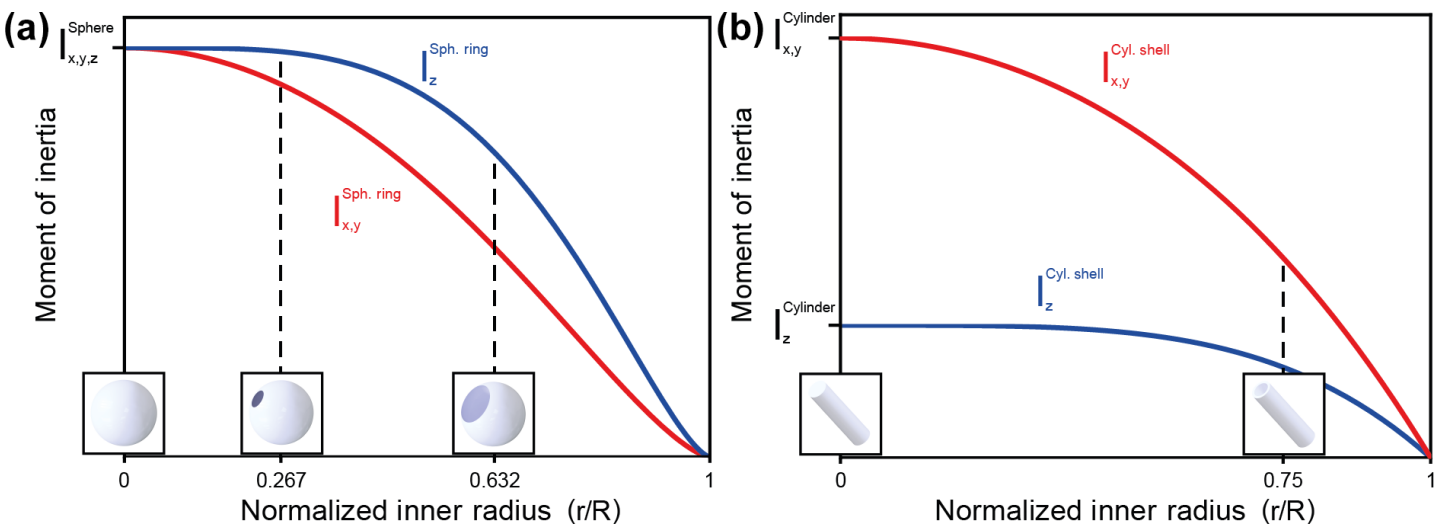

Figure 4. Moments of inertia for spherical and cylindrical rotors as a function of the normalized inner radius $r / R$. (a) $I_{z}$ (blue) and $I_{x, y}$ (red) for a spherical rotor as a function of the normalized inner radius. The rotors spun in this study correspond to $r / R=0.267$. The difference between $I_{z}$ and $I_{x, y}$ is maximized at $r / R=0.632$. (b) $I_{z}$ (blue) and $I_{x, y}$ (red) for a cylindrical rotor with aspect ratio $k=4$ as a function of the normalized inner radius.

inertia (Efroimsky, 2001, 2002; Krechetnikov and Marsden, 2007). This phenomenon has been observed in objects such as comets and asteroids as well as in early spacecraft such as Explorer 1 (Efroimsky, 2001; Krechetnikov and Marsden, 2007; Bracewell and Garriott, 1958). Explorer 1 was a cylindrically symmetric satellite with a high aspect ratio that was meant to spin about its axis of symmetry (the lowest inertia axis) but ended up developing a precession as it transitioned into an end-over-end spin (the highest inertia axis) as a result of energy being dissipated into the structure.

Due to dissipative interactions with the surrounding fluid, a high-aspect-ratio cylindrical MAS rotor cannot spin stably and continuously about its axis of symmetry without active stabilization (i.e., bearing), as its symmetry axis is also its lowest inertia axis. However, in the case of a spherical MAS rotor, the rotor is placed in the stator without a specific orientation and initially may spin about an arbitrary axis. As the axis of symmetry for a spherical ring is also its greatest inertia axis, a spherical rotor ultimately ends up stably spinning about its axis of symmetry due to rotational energy being dissipated by interactions with the gas stream and surrounding atmosphere. For this reason, a spherical rotor shows very stable on-axis spinning and resilience to crashing, as the rotor will always return to a stable minimum about its axis of symmetry after a perturbation.

Equations (1) and (2) suggest that a spherical rotor should spin most stably about its axis of symmetry, $z$, when $r / R=$ 0.632 , where the difference between $I_{z}$ and $I_{x, y}$ is maximized (Fig. 4a). Critically, this means that rotors with a spherical ring geometry can be quite stable even with very large sample volumes. When considering a packed rotor, as long as the density of the caps and sample is lower than the density of the rotor material, $I_{z}$ will be greater than $I_{x, y}$ for all values of $r / R$ between 0 and 1 , and stable on-axis spinning will occur. A detailed investigation of the moments of inertia for a packed spherical rotor taking into consideration the caps, sample, and rotor, each with different densities, can be found as an interactive notebook in the Supplement.

\section{Conclusions}

While turbine grooves can help to increase MAS rates for spherical rotors, the inertia tensor is responsible for its spinning stability. As MAS rates continue to increase to values in excess of $100 \mathrm{kHz}$, the possibility of spinning instabilities leading to rotor crashes becomes a significant concern. Spherical rotors spinning at high rates will be able to selfcorrect to a stable state after a perturbation due to the highest moment of inertia axis being aligned with the axis of symmetry. To achieve high MAS rates with spherical rotors, new rotors with smaller outer diameters must be designed and fabricated. These rotors could use shallow, Pelton-style grooves to increase the maximum spinning rate by about $30 \%$ compared to a rotor with no machined grooves, as observed here. However, since turbine grooves are not necessary to achieve stable spinning, these rotors could be fabricated without the need for complex micro-machining techniques to produce turbine grooves.

Code availability. The Mathematica code used to produce the plots seen in Fig. 4 as well as an analysis of the inertia tensor as a function of normalized inner radius for a packed spherical rotor with unique densities for the rotor, sample, and caps may be found in the Supplement.

Supplement. The supplement related to this article is available online at: https://doi.org/10.5194/mr-1-97-2020-supplement. 
Author contributions. TMOP performed the stator, rotor, and probe design, spin test and NMR data collection, the inertia tensor analysis, and the writing of the manuscript. AD and CG fabricated and installed the double-saddle coil and optical tachometer. PHC assisted with the design process, spin testing, and data collection. LEP assisted with 3D printing of the stators. NHA assisted with the rotor design process. ABB supervised the execution of experiments and guided the writing of the manuscript. All the authors contributed to the editing of the manuscript.

Competing interests. Alexander B. Barnes is an author on provisional patents related to this work filed by Washington University in Saint Louis (62/703,278 filed on 25 July 2018 and 62/672,840 filed on 17 May 2018). The authors declare that they have no conflict of interest.

Acknowledgements. This research was supported by an NIH Director's New Innovator Award (grant no. DP2GM119131) and startup funding from ETH Zürich. We thank Roland Walker for valuable assistance and advice with $3 \mathrm{D}$ printing.

Financial support. This research has been supported by the NIH Director's New Innovator Award (grant no. DP2GM119131).

Review statement. This paper was edited by Robert Tycko and reviewed by two anonymous referees.

\section{References}

Andrew, E. R.: Magic angle spinning, Int. Rev. Phys. Chem., 1, 195-224, https://doi.org/10.1080/01442358109353320, 1981.

Andrew, E. R., Bradbury, A., and Eades, R. G.: Removal of dipolar broadening of nuclear magnetic resonance spectra of solids by specimen rotation, Nature, 183, 1802-1803, https://doi.org/10.1038/1831802a0, 1959.

Bougault, C., Ayala, I., Vollmer, W., Simorre, J. P., and Schanda, P.: Studying intact bacterial peptidoglycan by proton-detected NMR spectroscopy at $100 \mathrm{kHz}$ MAS frequency, J. Struct. Biol., 206, 66-72, https://doi.org/10.1016/j.jsb.2018.07.009, 2019.

Bracewell, R. N. and Garriott, O. K.: Rotation of artificial Earth satellites, Nature, 182, 760-762, https://doi.org/10.1038/182760a0, 1958.

Cegelski, L., Kim, S. J., Hing, A. W., Studelska, D. R., O’Connor, R. D., Mehta, A. K., and Schaefer, J.: Rotational-echo double resonance characterization of the effects of vancomycin on cell wall synthesis in Staphylococcus aureus, Biochemistry, 41, 1305313058, https://doi.org/10.1021/bi0202326, 2002.

Chen, P., Albert, B. J., Gao, C., Alaniva, N., Price, L. E., Scott, F. J., Saliba, E. P., Sesti, E. L., Judge, P. T., Fisher, E. W., and Barnes, A. B.: Magic angle spinning spheres, Science Advances, 4, eaau1540, https://doi.org/10.1126/sciadv.aau1540, 2018.

Chen, P.-H., Gao, C., and Barnes, A. B.: Perspectives on microwave coupling into cylindrical and spherical rotors with dielectric lenses for magic angle spinning dy- namic nuclear polarization, J. Magn. Reson., 308, 106518, https://doi.org/10.1016/j.jmr.2019.07.005, 2019.

Clauss, J., Schmidt-Rohr, K., and Spiess, H. W.: Determination of domain sizes in heterogeneous polymers by solid-state NMR, Acta Polym., 44, 1-17, https://doi.org/10.1002/actp.1993.010440101, 1993.

Doty, F. D. and Ellis, P. D.: Design of high speed cylindrical NMR sample spinners, Rev. Sci. Instrum., 52, 1868-1875, https://doi.org/10.1063/1.1136530, 1981.

Efroimsky, M.: Relaxation of wobbling asteroids and comets Theoretical problems, perspectives of experimental observation, Planet. Space Sci., 49, 937-955, https://doi.org/10.1016/S00320633(01)00051-4, 2001.

Efroimsky, M.: Euler, Jacobi, and missions to comets and asteroids, Adv. Space Res., 29, 725-734, https://doi.org/10.1016/S02731177(02)00017-0, 2002.

Gao, C., Judge, P. T., Sesti, E. L., Price, L. E., Alaniva, N., Saliba, E. P., Albert, B. J., Soper, N. J., Chen, P. H., and Barnes, A. B.: Four millimeter spherical rotors spinning at $28 \mathrm{kHz}$ with doublesaddle coils for cross polarization NMR, J. Magn. Reson., 303, 1-6, https://doi.org/10.1016/j.jmr.2019.03.006, 2019.

Knight, M. J., Pell, A. J., Bertini, I., Felli, I. C., Gonnelli, L., Pierattelli, R., Herrmann, T., Emsley, L., and Pintacuda, G.: Structure and backbone dynamics of a microcrystalline metalloprotein by solid-state NMR, P. Natl. Acad. Sci. USA, 109, 11095-11100, https://doi.org/10.1073/pnas.1204515109, 2012.

Kong, X., Deng, H., Yan, F., Kim, J., Swisher, J. A., Smit, B., Yaghi, O. M., and Reimer, J. A.: Mapping of functional groups in metal-organic frameworks, Science, 341, 882-885, https://doi.org/10.1126/science.1238339, 2013.

Krechetnikov, R. and Marsden, J. E.: Dissipation-induced instabilities in finite dimensions, Rev. Mod. Phys., 79, 519-553, https://doi.org/10.1103/RevModPhys.79.519, 2007.

Lesage, A., Gardiennet, C., Loquet, A., Verel, R., Pintacuda, G., Emsley, L., Meier, B. H., and Böckmann, A.: Polarization transfer over the water-protein interface in solids, Angew. Chem. Int. Edit., 47, 5851-5854, https://doi.org/10.1002/anie.200801110, 2008.

Lowe, I. J.: Free induction decays of rotating solids, Phys. Rev. Lett., 2, 285-287, https://doi.org/10.1103/PhysRevLett.2.285, 1959.

McDermott, A.: Structure and Dynamics of Membrane Proteins by Magic Angle Spinning SolidState NMR, Ann. Rev. Biophys., 38, 385-403, https://doi.org/10.1146/annurev.biophys.050708.133719, 2009.

Narayanan, P., Lefcourt, A. M., Tasch, U., Rostamian, R., Grinblat, A., and Kim, M. S.: Theoretical analysis of stability of axially symmetric rotating objects with regard to orienting apples, T. ASABE, 51, 1353-1364, https://doi.org/10.13031/2013.25218, 2008.

Petkova, A. T., Leapman, R. D., Guo, Z., and Yau, W.-M.: SelfPropagating, Molecular-Level Polymorphism in Alzheimer's $\beta$ Amyloid Fibrils, Science, 307, 262-266, 2005.

Retel, J. S., Nieuwkoop, A. J., Hiller, M., Higman, V. A., BarbetMassin, E., Stanek, J., Andreas, L. B., Franks, W. T., Van Rossum, B. J., Vinothkumar, K. R., Handel, L., De Palma, G. G., Bardiaux, B., Pintacuda, G., Emsley, L., Kühlbrandt, W., and Oschkinat, H.: Structure of outer membrane protein $\mathrm{G}$ in lipid bi- 
layers, Nat. Commun., 8, 2073, https://doi.org/10.1038/s41467017-02228-2, 2017.

Schaefer, J. and Stejskal, E. O.: Carbon-13 Nuclear Magnetic Resonance of Polymers Spinning at the Magic Angle, J. Am. Chem. Soc., 98, 1031-1032, https://doi.org/10.1021/ja00420a036, 1976.

Theint, T., Nadaud, P. S., Aucoin, D., Helmus, J. J., Pondaven, S. P., Surewicz, K., Surewicz, W. K., and Jaroniec, C. P.: Species-dependent structural polymorphism of Y145Stop prion protein amyloid revealed by solid-state NMR spectroscopy, Nat. Commun., 8, 753, https://doi.org/10.1038/s41467-017-00794-z, 2017.

Thornton, S. T. and Marion, J. B.: Classical Dynamics of Particles and Systems, Brooks/Cole, 2004.
Trebosc, J., Wiench, J. W., Huh, S., Lin, V. S., and Pruski, M.: Studies of organically functionalized mesoporous silicas using heteronuclear solid-state correlation NMR spectroscopy under fast magic angle spinning, J. Am. Chem. Soc., 127, 7587-7593, https://doi.org/10.1021/ja0509127, 2005.

Wang, S., Munro, R. A., Shi, L., Kawamura, I., Okitsu, T., Wada, A., Kim, S. Y., Jung, K. H., Brown, L. S., and Ladizhansky, V.: Solid-state NMR spectroscopy structure determination of a lipid-embedded heptahelical membrane protein, Nat. Methods, 10, 1007-1012, https://doi.org/10.1038/nmeth.2635, 2013.

Wilhelm, D., Purea, A., and Engelke, F.: Fluid flow dynamics in MAS systems, J. Magn. Reson., 257, 51-63, https://doi.org/10.1016/j.jmr.2015.05.006, 2015. 